\title{
Hematological and Immunological Studies on the Effect of Hepatitis B Virus Vaccination in Hepatitis and Non-Hepatitis, Iron Chelating Dependent or Independent Egyptian Thalassemia Patients Eman G. Helal*, Ali F.M. El-Sayed**, Noran Abu- Ouf*, Nahla G. Mohamed** and Mona A.M. Ahmed** \\ *Al-Azhar University, Faculty of Science (Girls) and ** Virology Sector Research Unit, VACSERA. Egypt.
}

\begin{abstract}
Background: Regular transfusion in thalassemia major patients increases life expectancy and improves quality of life. Blood transfusion is the main sources for viral transmission to Thalassemia patients. So, detection of viral antigens using more than one technique must be adopted. Iron and its binding proteins have immune regulatory properties and shifting of immune regulatory balance by iron excess or deficiency may produce severe deleterious physiological effects. Thus, the aim of this study was to assess the efficacy of immunization and determine the immune response of beta-thalassemia patients. Also, to evaluate the effects of iron overload chelating therapy and hepatitis B virus (HBV) vaccination on some immunological and hematological parameters in hepatitis and non hepatitis Egyptian thalassemia patients.

Methods: Forty homozygote Thalassemia patients attending blood bank, therapeutic unite, Holding company for Biological production and Vaccine, VACSERA were chosen for this study (age range 4-30years, mean 14 years, 18 females $46 \%$ and 22 males $54 \%$ ).

Results: There was no significant correlation between $\mathrm{HBs} \mathrm{Ab}$ level in control and non vaccinated groups that include Thalassemia, hepatitis, non hepatitis, either iron chelating therapy dependent or independent patients. In the same time, there was no significant correlation between Ab level in vaccinated control and Thalassemia groups. $\mathrm{HbF}$ and $\mathrm{HbA} 2 \%$ showed significant and highly significant increases respectively, in most of groups especially, Thalassemia, hepatitis and iron chelating independent, vaccinated or non vaccinated groups. While $\mathrm{HbA}$ may be present in small amount or completely absent. RBCs count, $\mathrm{Hb} \%$, Hematocrit and MCV values were decreased significantly in all patient's groups vaccinated or non vaccinated compared to control group, while $\mathrm{MCH}$ and $\mathrm{MCHC}$ were not changed in patient' groups compared to control group. Platelets count was increased significantly in most patient' groups (except non vaccinated, Thalassemia, hepatitis, iron chelating dependent) compared to control group. Also, WBCs count was increased significantly in most groups (except non vaccinated, Thalassemia, hepatitis or non hepatitis, iron chelating dependent or independent) in comparison with the control group. All patients (hepatitis, vaccinated or non vaccinated) had high significant increase in gamma globulins compared to control group.

Conclusion: Hematological and immunological measurements for hepatitis Thalassemia patients are important to monitor and treat the disease.
\end{abstract}

Key words: Blood transfusion, Thalassemia patients, iron excess, HBs, gamma globulins.

\section{Introduction:}

Thalassemia ( is also known as Cooley's anemia) is a group of inherited hemoglobin disorders characterized by reduced synthesis of one or more of the globin chains leading to imbalanced globin synthesis which is the major factor in determining the severity of the disease in the Thalassemia syndromes. In Egypt, $\beta$ thalassemia is the commonest cause of chronic hemolytic anemia and it represents a major genetic disease and a public health problem which engulfs a large portion of the country's health financial

plan $^{(1,2)}$ and the rate of new birth with Thalassemia per year is $1000 / 1.5$ million $^{(3)}$.
The thalassemias are classified according to which chain of the hemoglobin molecule is affected. In $\alpha$ - thalassemias, production of the $\alpha$ globin chain is affected, while in $\beta$ - thalassemia production of the $\beta$ globin chain is affected. Thalassemia is the most common type of hemoglobinopathy transmitted by heredity. The decrease or loss of $\alpha$ or $\beta$ chain has unfavorable effects on the production and the survival of red blood cells (RBCs) and may cause a decrease in the concentration of the globin chain and of hemoglobin, resulting in microcytosis and hypochromia ${ }^{(4)}$.

The management of thalassemia major essentially comprises of regular "safe blood transfusion" and a life long iron-chelation 
therapy ${ }^{(5)}$. HCV infection has gained importance particularly as one of the major complications in multiply transfused patients during the last decade. This is especially true for countries where $\mathrm{HCV}$ is more prevalent in general population and therefore also amongst blood donors. The prevalence rate of seropositivity increases with the number of transfusions ${ }^{(6,7,8)}$. This post-transfusion hepatitis has significantly contributed to morbidity in thalassemia ${ }^{(9)}$. It should be remembered that $\mathrm{HCV}$ hepatitis is more threatening than HBV hepatitis due to a greater risk of chronic liver disease such as cirrhosis and hepatocellular carcinoma ${ }^{(10)}$.

In case of hepatitis B, since an effective vaccine is available, immunization against this virus before transfusion management is started which could be effectively protected against transfusion hepatitis B. However, since no such vaccine is so far available against hepatitis $C$, the only effective protective measure against this virus is provision of $\mathrm{HCV}$ negative blood for transfusion. Therefore, screening of transfused blood for HCV in not only mandatory, but also it is essential to use the most sensitive screening methods with least possible false-negative results (7).

Transfused antibodies may inhibit the recipient's sensitization and primary immune response to the homologous antigen, especially when the antibody level in the transferred blood is high whereas the secondary immune response is not affected ${ }^{(11)}$.

Fetal hemoglobin $(\mathrm{HbF})$ is the main hemoglobin component throughout fetal life and at birth, accounting for approximately $80 \%$ of total hemoglobin in newborns. $\mathrm{HbF}$ is produced from the sixth week of gestation and during the rest of fetal life, replacing the embryonic hemoglobins ${ }^{(12)}$. After birth, HbF synthesis rapidly declines and $\mathrm{HbF}$ is gradually substituted by adult hemoglobin ( $\mathrm{HbA}$ ) in the peripheral blood, so that within the first two years of life, the characteristic hemoglobin phenotype of the adult (96-98\%) with very low levels of $\mathrm{HbF}$ (less than $1 \%$ ) is found ${ }^{(13)}$ and most of the remainder is structurally different hemoglobin called hemoglobin $\mathrm{A}_{2}\left(\mathrm{HbA}_{2}\right)^{(14)}$. HbF measurement is clinically useful in the study and diagnosis of some important globin gene disorders where $\mathrm{HbF}$ levels may vary considerably (mainly, $\beta$ and $\delta \beta$-thalassemia) ${ }^{(12)}$.

Iron and its binding proteins have immunoregulatory properties, and shifting of immunoregulatory balances by iron excess or deficiency may produce severe, deleterious physiological effects. Effects of iron overload include decreased antibody- mediated and mitogen- stimulated phagocytosis by monocytes and macrophages, alterations in T- lymphocyte subsets and modification of lymphocyte distribution in different compartments of the immune system which is associated with $\mathrm{HCV}$ positively ${ }^{(15)}$. The poor ability of lymphocytes to sequester excess iron in ferritin may help to explain the immune system abnormalities in iron overload patients ${ }^{(16)}$. In patients with choronic hepatitis (CHC) with no other cause for iron overload, iron may be a cofactor in the development of liver injury and correlate with disease severity ${ }^{(17)}$.

Since iron overload and hepatitis $\mathrm{C}$ virus $(\mathrm{HCV})$ are the main causes of chronic liver diseases in $\beta$ - Thalassemia patients. The aim of this study was to evaluate the effects of iron overload chelating therapy and hepatitis $B$ virus (HBV) vaccination on some immunological and hematological parameters in hepatitis and non hepatitis Egyptian Thalassemia patients.

\section{Material and Methods:}

Study subjects included 40 patients with age range 4-30years (mean 14 years), 18 females $46 \%$ and 22 males $54 \%$. All patients were Thalassemia Major. Every patient received approximately blood transfusion (3- 4 week intervals) the range of hemoglobin was 6.5$7.5 \mathrm{~g} / \mathrm{dl}$. The samples were collected before the blood transfusion.

Twenty patients of all were positive hepatitis $\mathrm{C}$ virus (HCV). These 20 patients were divided into 2 subgroups, $50 \%$ of them used iron chelating therapy (iron chelating dependent) and the other $50 \%$ were iron chelating independent. $50 \%$ of every subgroup were vaccinated with HBV. Other 20 (non HCV infection) also divided to 2 subgroups, $50 \%$ of them used iron chelating therapy (iron chelating dependent) and the other $50 \%$ were iron chelating independent. $50 \%$ of every subgroup were vaccinated with HBV. In addition 10 healthy individuals 4 females (40\%) and 6 males $(60 \%)$ were enrolled in the study to act as a reference group. The control group was divided into 2 subgroups (5 of them were $\mathrm{HBV}$ vaccinated and 5 were non vaccinated).

A-Immunological parameters;

1- Rapid detection for HCV Ab:

Qualitative detection of $\mathrm{HCV}$ antibodies ( $\mathrm{HCV} \mathrm{Ab}$ ) were carried out by the HCV rapid screen test (RST) according to Acon comp, USA. Briefly, RST is a chromatographic immunoassay for detection of antibodies of 
hepatitis in human serum or plasma. HCV recombinant antigens are precoated on to membrane as a capture reagent to specific antibodies $(\mathrm{Ab})$ on the test region.

2- Rapid detection for HBs Ag:

RST for direct qualitative detection of antibodies to hepatitis B surface antigen (HBs $\mathrm{Ag}$ ) is precoated on to membrane as a capture reagent content on the test region. Specimen is allowed to react with colloidal gold particales, which have been labeled with other specific antibodies.

3- Determination of HBs Ab by Axyme radioimmunoassay :

HBs $\mathrm{Ab}$ was determined by using an automated instrument model (ER-HYD-1525 199) closed system, according to (Abbott laboratories diagnostic division, operations manual, volume 1997, USA).

4- Detection of hemoglobin $\mathrm{A}_{2}$ and $\mathrm{F}$ $\left(\mathrm{HbA}_{2} / \mathrm{F}\right)$ variants by HPLC:

$\mathrm{HbA} 2 / \mathrm{F}$ were achieved by using technical hemoglobin test High Performance Liquid Chromatography (HPLC) system $\mathrm{HbA}_{2} / \mathrm{F}$ according to ${ }^{(18)}$.

B- Hematological parameters:

Blood counts were done by coulter counter AC-T8 which include red blood cells count (RBCs) count $\left(\times 10^{6} / \mathrm{cm}^{3}\right)$; hemoglobin concentration ( $\mathrm{Hb} \mathrm{g} / 100 \mathrm{ml})$, hematocrit (\%); $\mathrm{RBCs}$ indices (MCV, $\mathrm{MCH}$ and $\mathrm{MCHC}$ ); total leukocyte count $\left(\mathrm{x}^{3} 0^{3} / \mathrm{cm}^{3}\right)$ and platelet count $\left(\mathrm{x} 10^{3} / \mathrm{cmm}\right)$. Also; smears of blood stained with Leishman's stain were prepared for differential leukocyte count according to Dacie and Lewis (19). Blood slides were also examined to detect variations in structure, size, shape and content of RBCs ${ }^{(20)}$.

Statistical analysis was carried out using SAS program (SAS, 1988). Student's t-test was run to test the effect of vaccination within each treatment. One way analysis of variance
(ANOVA) followed by Duncan's multiple range test were used to test the effect of treatments on different measurements in non vaccinated and vaccinated groups. Cross tabulation and Chi square analysis were used to compare the prevalence of HBs between different groups.

\section{Results:}

\section{Immunological parameters changes:}

In the present study, the HBv antibody level was detected in Thallassimia patients and it was noted that the Ab level in sera of patients $(100 \%)$ ranged from $(0-100) \mathrm{mIU} / \mathrm{ml}$. Immune response of patients was classified according to the $\mathrm{Ab}$ level as follow: (0-10 $\mathrm{mIU} / \mathrm{ml}$ ) is -ve immune response or nonvaccinated and it was detected in $40 \%$ of non vaccinated groups (thalassemic, non hepatitis, iron chelating independent); $50 \%$ of non vaccinated group were positively reacting to the vaccine but recommended to be revaccinated; the $\mathrm{Ab}$ level was $(10-100 \mathrm{mIU} / \mathrm{ml})$ and the rest $50 \%$ were + ve reacting to the vaccine. There was a significant correlation between $\mathrm{ABv} A b$ level and non vaccinated patient groups (table 1).

The recorded data revealed that there was no significant correlation between $\mathrm{Ab}$ level in control and non vaccinated groups that include Thalassemia, hepatitis, non hepatitis, either iron chelating therapy dependent or independent patients. In the same time, there was no significant correlation between $\mathrm{Ab}$ level in vaccinated control and Thalassemia groups.(table, 1 ).

It is noticed that, serum ferritin level in all patient groups (non vaccinated and vaccinated) was elevated significantly $(\mathrm{P} \leq 0.001)$ compared to control groups. On the other hand, vaccinated control group had decreased serum ferritin copared to non vaccinated control group. The highest serum ferritin level was detected in vaccinated, Thalassemia, non hepatitis, iron chelating compared to other patient groups. 
Table (1): Assessment of hepatitis B surface antibody level(\%) and ferritin $(\mathrm{ng} / \mathrm{mL})$ i $n$ the sera of Thalassemia patients in relation to control groups

\begin{tabular}{|c|c|c|c|c|c|c|c|}
\hline \multirow{3}{*}{ Group } & & \multicolumn{4}{|c|}{$\begin{array}{l}\text { Hepatitis B surface } \\
\text { antibody level }\end{array}$} & \multicolumn{2}{|l|}{ Ferritin } \\
\hline & & \multicolumn{2}{|c|}{$\begin{array}{l}\text { Non } \\
\text { vaccinated }\end{array}$} & \multicolumn{2}{|c|}{ vaccinated } & $\begin{array}{l}\text { Non } \\
\text { vaccinated }\end{array}$ & vaccinated \\
\hline & HBs $\mathrm{Ab}$ titer & $\%$ & & $\%$ & & & \\
\hline \multirow{3}{*}{ Control group. } & Negative $(\leq 10)$ & 40 & & 20 & & \multirow{3}{*}{$\begin{array}{l}148.8 \pm \\
5.817\end{array}$} & \multirow{3}{*}{$\begin{array}{l}112.0 \pm \\
3.661 * * *\end{array}$} \\
\hline & $\begin{array}{l}\text { Revaccinated (10- } \\
>100)\end{array}$ & 60 & & 40 & & & \\
\hline & $\begin{array}{l}\text { Immune response } \\
(\geq 100)\end{array}$ & \multicolumn{2}{|l|}{0} & 40 & & & \\
\hline \multirow{3}{*}{$\begin{array}{l}\text { Thalassemic, hepatitis, } \\
\text { iron chelating } \\
\text { dependent group }\end{array}$} & Negative $(\leq 10)$ & 60 & \multirow{3}{*}{$*$} & 0 & & \multirow{3}{*}{$\begin{array}{l}2737.0 \pm \\
140.941 * * *\end{array}$} & \multirow{3}{*}{$\begin{array}{l}2454.6 \pm \\
117.269^{* * *}\end{array}$} \\
\hline & $\begin{array}{l}\text { Revaccinated (10- } \\
\geq 100)\end{array}$ & 20 & & 60 & & & \\
\hline & $\begin{array}{l}\text { Immune response } \\
(\geq 100)\end{array}$ & 20 & & 40 & & & \\
\hline \multirow{3}{*}{$\begin{array}{l}\text { Thalassemic, hepatitis, } \\
\text { iron chelating } \\
\text { independent group }\end{array}$} & Negative $(\leq 10)$ & 60 & \multirow{3}{*}{$*$} & 20 & & \multirow{3}{*}{$\begin{array}{l}2274.2 \pm \\
128.716^{* * *}\end{array}$} & \multirow{3}{*}{$\begin{array}{l}1624.6 \pm \\
115.017^{* * *}\end{array}$} \\
\hline & $\begin{array}{l}\text { Revaccinated (10- } \\
\geq 100)\end{array}$ & 20 & & 60 & & & \\
\hline & $\begin{array}{l}\text { Immune response } \\
(\geq 100)\end{array}$ & 20 & & 20 & & & \\
\hline \multirow{3}{*}{$\begin{array}{l}\text { Thalassemic, non } \\
\text { hepatitis, iron chelating } \\
\text { dependent group. }\end{array}$} & Negative $(\leq 10)$ & 40 & \multirow{3}{*}{$*$} & 40 & & \multirow{3}{*}{$\begin{array}{l}1501 \pm \\
162.984 * * *\end{array}$} & \multirow{3}{*}{$\begin{array}{l}3223.0 \pm \\
104.845 * * *\end{array}$} \\
\hline & $\begin{array}{l}\text { Revaccinated (10- } \\
\geq 100)\end{array}$ & 20 & & 20 & & & \\
\hline & $\begin{array}{l}\text { Immune response } \\
(\geq 100)\end{array}$ & 40 & & 40 & & & \\
\hline \multirow{3}{*}{$\begin{array}{l}\text { Thalassemia, non } \\
\text { hepatitis, iron chelating } \\
\text { independent group }\end{array}$} & Negative $(\leq 10)$ & 50 & \multirow{3}{*}{$*$} & 0 & \multirow{3}{*}{$*$} & \multirow{3}{*}{$\begin{array}{l}2411.0 \pm \\
184.806\end{array}$} & \multirow{3}{*}{$\begin{array}{l}2208.8 \pm \\
100.751\end{array}$} \\
\hline & $\begin{array}{l}\text { Revaccinated (10- } \\
\geq 100)\end{array}$ & 25 & & 100 & & & \\
\hline & $\begin{array}{l}\text { Immune response } \\
(\geq 100)\end{array}$ & 25 & & 0 & & & \\
\hline
\end{tabular}

Ferritin results are $\mathrm{M} \pm \mathrm{S}$.E. of 5 patients.

* Significant at $\mathrm{P} \leq 0.05$ compared to control groups.

$* * *$ Significant at $\mathrm{P} \leq 0.001$ compared to control groups.

In the present study, the $\mathrm{HbF}$ was $100 \%$ in all Thalassemia patients. $\mathrm{HBF}$ were $0.1 \%$ and $0.08 \%$ in control non vaccinated and vaccinated control group respectively, on the other hand, thes percentages were increased up to $12.6 \%$ in vaccinated Thalassemia, non hepatitis, iron chelating independent and to $41.5 \%$ in vaccinated Thalassemia,hepatitis, iron chelating independent patients. There was a significant correlation between $\mathrm{HbF}$ values in non vaccinated (Thallassimia, hepatitis, iron chelating independent group and Thallassimia, nonhepatitis, iron chelating therapy dependent group). Also, a non significant correlation between Thallassimia, hepatitis, iron chelating therapy dependent group was detected (table 2 ). On the other hand, in vaccinated groups a highly significant correlation between Thallassimia, nonhepatitis, iron chelating therapy dependent and independent and control groups. Also, a non significant correlation between Thallassimia, hepatitis, iron chelating therapy dependent and Thallassimia, nonhepatitis, iron chelating therapy dependent groups were detected (table 2).

Regarding the vaccination effect on $\mathrm{HbF}$ values, the non vaccinated patient group tend to have lower $\mathrm{HbF} \%$ than vaccinated one, the difference was significantly increased ( $\mathrm{P}$ $\leq 0.05$ ) in Thallassimia, non hepatitis, iron chelating therapy dependent or independent, while other groups showed non significant changes compared to vaccinated groups (table 2 and plate1 pictures 1-5).

In this study, the $\mathrm{HbA}_{2} \%$ in non vaccinated group showed significant increase value in comparison with the control group, while $\mathrm{HbA} 2 \%$ value in Thalassemia, hepatitis and iron chelating therapy independent patients was significantly higher than its values in Thalassemia, non hepatitis patients either iron chelating therapy dependent or independent. 
Similarly, $\mathrm{HbA} 2 \%$ value in the last two groups was no significant related to its values in Thalassimia, hepatitis and iron chelating therapy dependent patients. While in vaccinated groups significant correlative values of $\mathrm{HbA} 2 \%$ were noted compare to control groups (table2 and plate1 pictures 1-5).

Table (2): Comparative evaluation of $\mathrm{HbA}_{2} \%$ and $\mathrm{HbF} \%$ variants in Thalassemia patients in relation to control groups.

\begin{tabular}{|c|c|c|c|c|c|c|}
\hline \multirow[b]{2}{*}{ Groups } & \multicolumn{2}{|l|}{$\mathrm{HbA} 2 \%$} & \multicolumn{2}{|l|}{$\mathrm{HbF} \%$} & \multicolumn{2}{|l|}{$\mathrm{HbA} \%$} \\
\hline & $\begin{array}{l}\text { Non } \\
\text { vaccinat } \\
\text { ed }\end{array}$ & $\begin{array}{l}\text { Vaccinat } \\
\text { ed }\end{array}$ & $\begin{array}{l}\text { Non } \\
\text { vaccinat } \\
\text { ed }\end{array}$ & $\begin{array}{l}\text { Vaccinat } \\
\text { ed }\end{array}$ & $\begin{array}{l}\text { Non } \\
\text { vaccinat } \\
\text { ed }\end{array}$ & $\begin{array}{l}\text { Vaccinat } \\
\text { ed }\end{array}$ \\
\hline Control group & $\begin{array}{l}\text { 2.180 } \pm \\
0.097\end{array}$ & $\begin{array}{l}2.380 \pm \\
\mathbf{0 . 0 8 0}\end{array}$ & $\begin{array}{l}0.100 \pm \\
0.063\end{array}$ & $\begin{array}{l}0.080 \pm \\
0.049\end{array}$ & $\begin{array}{l}83.980 \pm \\
1.474\end{array}$ & $\begin{array}{l}83.420 \pm \\
0.881\end{array}$ \\
\hline $\begin{array}{l}\text { Thalassemic, } \\
\text { hepatitis, iron } \\
\text { chelating dependent }\end{array}$ & $\begin{array}{l}7.200 \pm \\
0.679 *\end{array}$ & $\begin{array}{l}\text { 5.140 } \pm \\
0.623^{*}\end{array}$ & $\begin{array}{l}19.740 \pm \\
3.718^{*}\end{array}$ & $\begin{array}{l}\text { 20.56 } \pm \\
\text { 02.096* }\end{array}$ & $\begin{array}{l}\text { 71.000士 } \\
2.816^{*}\end{array}$ & $\begin{array}{l}\text { 69.86士 } \\
2.484 *\end{array}$ \\
\hline $\begin{array}{l}\text { Thalassemic,hepatitis, } \\
\text { iron chelating } \\
\text { independent }\end{array}$ & $\begin{array}{l}9.660 \pm \\
0.344^{*}\end{array}$ & $\begin{array}{l}7.180 \pm \\
0.689 * a\end{array}$ & $\begin{array}{l}35.860 \pm \\
1.904 * *\end{array}$ & $\begin{array}{l}41.580 \pm \\
5.209 * *\end{array}$ & $\begin{array}{l}69.660 \pm \\
2.267^{*}\end{array}$ & $\begin{array}{l}60.440 \pm \\
0.703 * *\end{array}$ \\
\hline $\begin{array}{l}\text { Thalassemic, non } \\
\text { hepatitis, iron } \\
\text { chelating dependent }\end{array}$ & $\begin{array}{l}\text { 7.940 } \pm \\
0.469^{*}\end{array}$ & $\begin{array}{l}7.780 \pm 0 . \\
629 *\end{array}$ & $\begin{array}{l}\text { 18.740 } \pm \\
0.841 *\end{array}$ & $\begin{array}{l}15.20 \pm \\
01.159 * *\end{array}$ & $\begin{array}{l}69.560 \pm \\
2.494 *\end{array}$ & $\begin{array}{l}\text { 74.260 } \pm \\
3.799 *\end{array}$ \\
\hline $\begin{array}{l}\text { Thalassemic, non } \\
\text { hepatitis, iron } \\
\text { chelating independent }\end{array}$ & $\begin{array}{l}9.225 \pm \\
0.549 * *\end{array}$ & $\begin{array}{l}7.467 \pm \\
0.249 * a\end{array}$ & $\begin{array}{l}22.475 \pm \\
2.089\end{array}$ & $\begin{array}{l}12.617 \pm 0 \\
.695 * *\end{array}$ & $\begin{array}{l}\text { 65.050 } \pm \\
3.401 *\end{array}$ & $\begin{array}{l}\text { 70.983 } \pm \\
\text { 3.205* }\end{array}$ \\
\hline
\end{tabular}

Mean of 5 patients/ group \pm S.E.

$*$ = significant at $\mathrm{p} \leq 0.05$ compared to control group.

$* *=$ significant at $\mathrm{p} \leq 0.01$ compared to control group.

a significant at $\mathrm{P} \leq 0.05$ compared to non vaccinated similar groups.

Regarding the vaccination effect on $\mathrm{HbA}_{2} \%$, the vaccinated patient group tended to have lower $\mathrm{HbA}_{2} \%$ than non vaccinated one, the difference was significantly decreased $(\mathrm{P} \leq$ 0.05 ) in Thallassimia, iron chelating therapy independent either with or without hepatitis. While, other groups showed non significant changes compared to vaccinated groups (table 2).

Table 2 represents the changes in $\mathrm{HbA} \%$ in different patients groups. $\mathrm{HbA} \%$ was decreased in all patient groups $(\mathrm{P} \leq 0.05)$ compared to control group. In both non vaccinated and vaccinated groups, $\mathrm{HbA} \%$ showed non significant differences between Thalassimia, hepatitis or non hepatitis either chelating therapy dependent or independent. The lowest $\mathrm{HbA} \%$ was recorded in vaccinated, Thalassimia, hepatitis, iron chelating independent patients (table 2). 


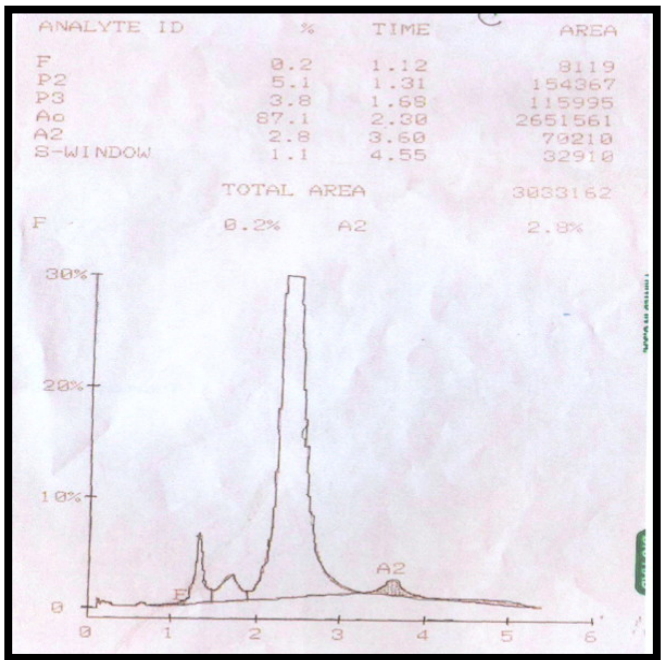

Picture (1): Control group

$\mathrm{HbF}=0.2 \%$

$\mathrm{HbA}=2.8 \%$

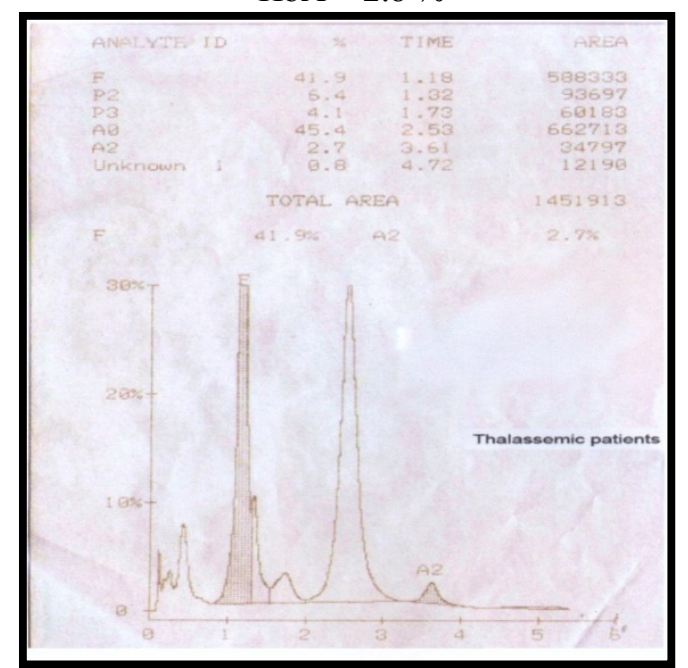

Picture (2): Thalassemia, non hepatic, iron chelating independent group

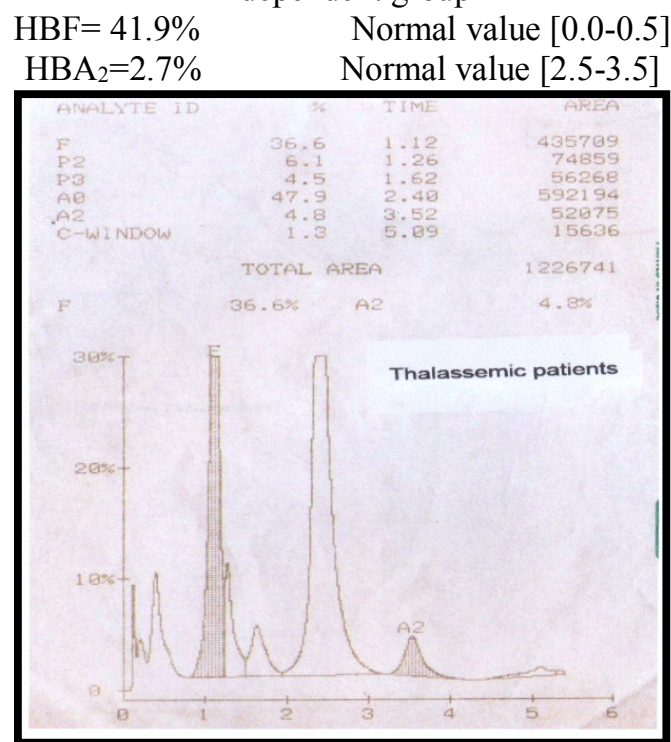

Picture (4): Thalassemia, hepatic, iron chelating dependent group $\mathrm{HBF}=36.6 \% \quad$ Normal value $[0.0-0.5]$ $\mathrm{HBA}_{2}=4.8 \% \quad$ Normal value [2.5-3.5]
Normal value $[0.0-0.5]$

Normal value $[2.5-3.5]$

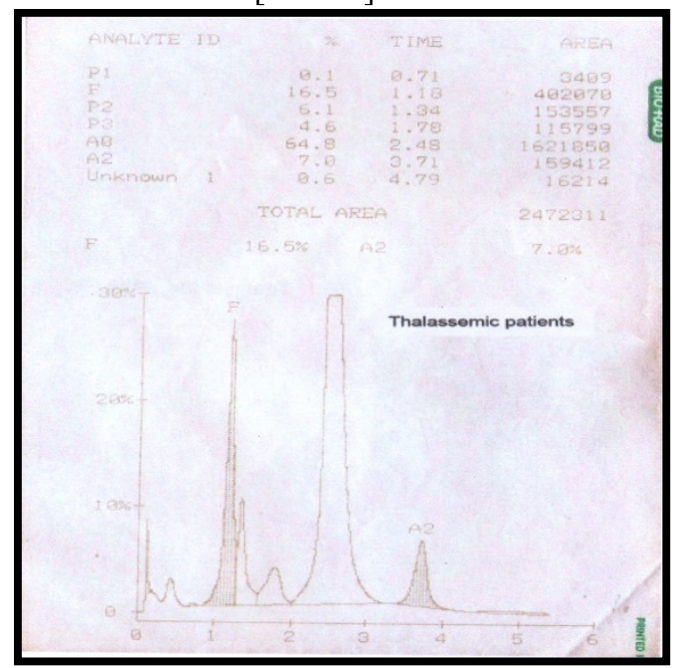

Picture (3): Thalassemia, non hepatic, iron chelating dependent group

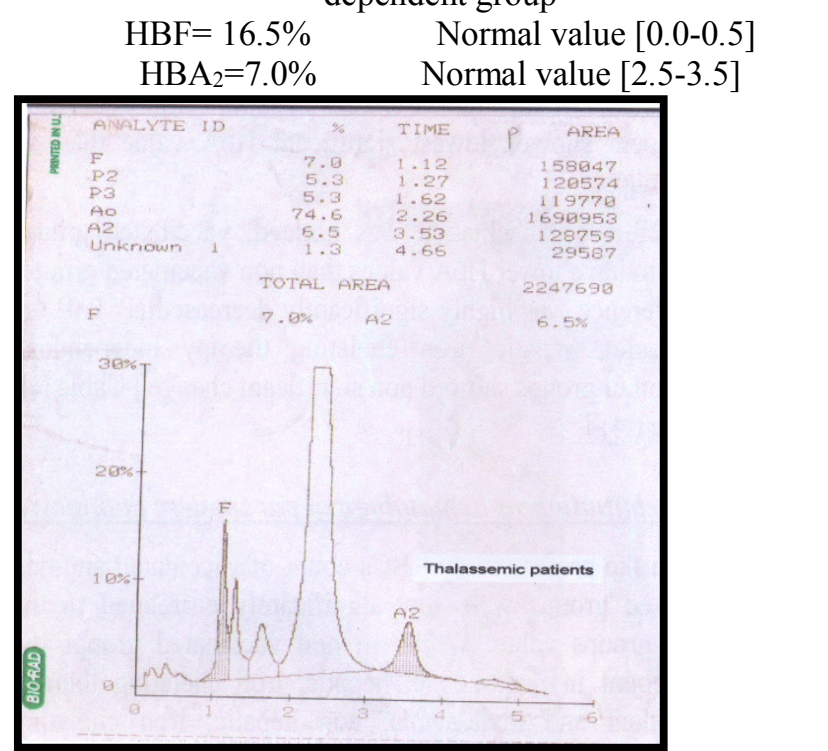

Picture (5): Thalassemia, non hepatic, iron chelating dependent group

$$
\begin{array}{ll}
\mathrm{HBF}=7.0 \% & \text { Normal value }[0.0-0.5] \\
\mathrm{HBA}_{2}=6.5 \% & \text { Normal value }[2.5-3.5]
\end{array}
$$


Plate (1) HPLC hemoglobin variants to evaluate $\mathrm{HbF} \& \mathrm{HBA}_{2}$

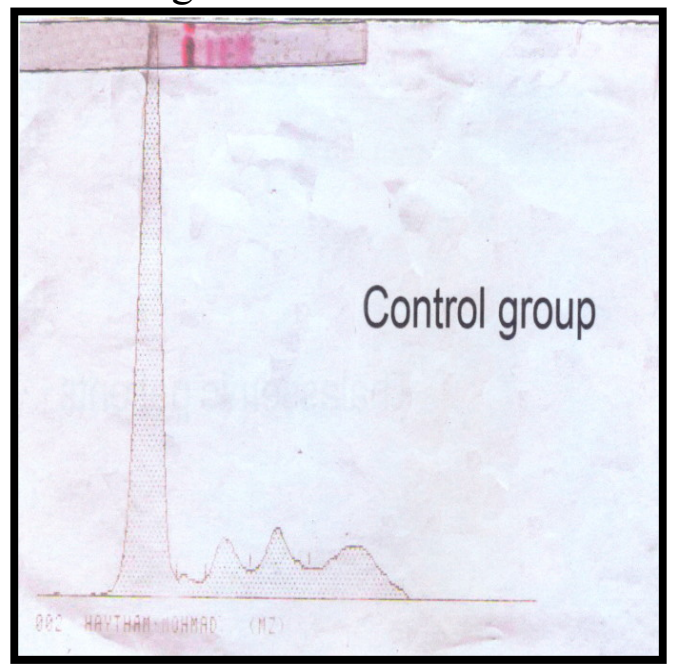

Picture (1): Control group

$\alpha_{1}=2.2,1.5-4.5 \% \quad ; \quad \alpha_{2}=7.8,6-12 \% ; \beta=11.2,11-17 \% \quad \gamma=14.7,11-19 \%$

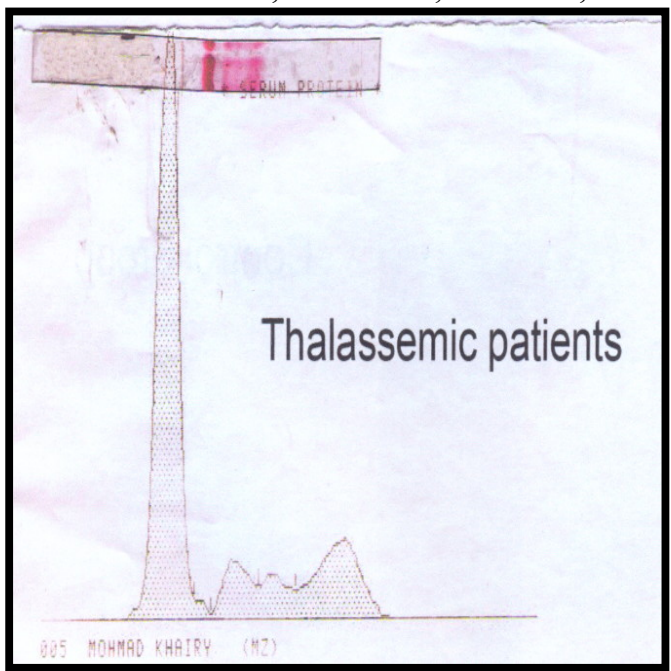

Picture (2): Thalassemia, non hepatic , iron chelating dependent $\alpha_{1}=1.1,1.5-4.5 \%$ $\alpha_{2}=8.3,6-12 \%$ $\beta=6.0,11-19 \%$ $\gamma=18.1,11-19 \%$

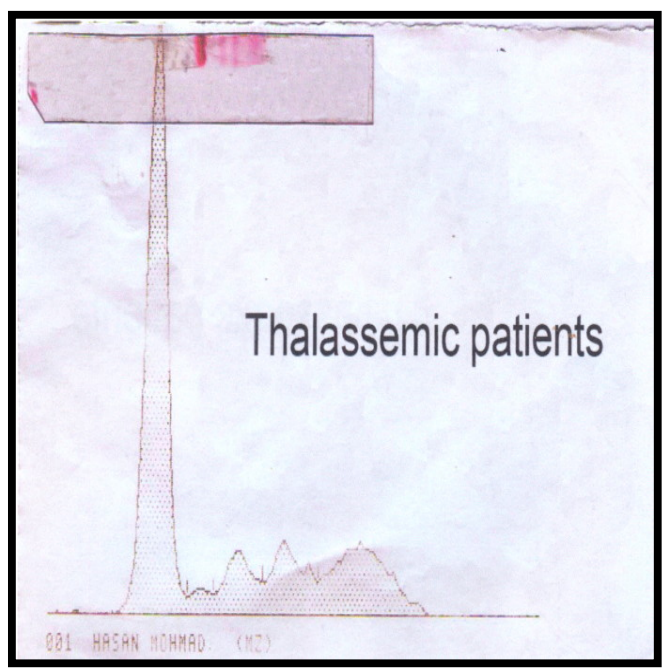

Picture (4): Thalassemia, hepatic , iron chelating independent $\alpha_{1}=2.4,1.5-4.5 \%$ $\beta=11.4,11-19 \%$

$$
\alpha_{2}=8.5,6-12 \%
$$$$
\gamma=19.5,11-19 \%
$$

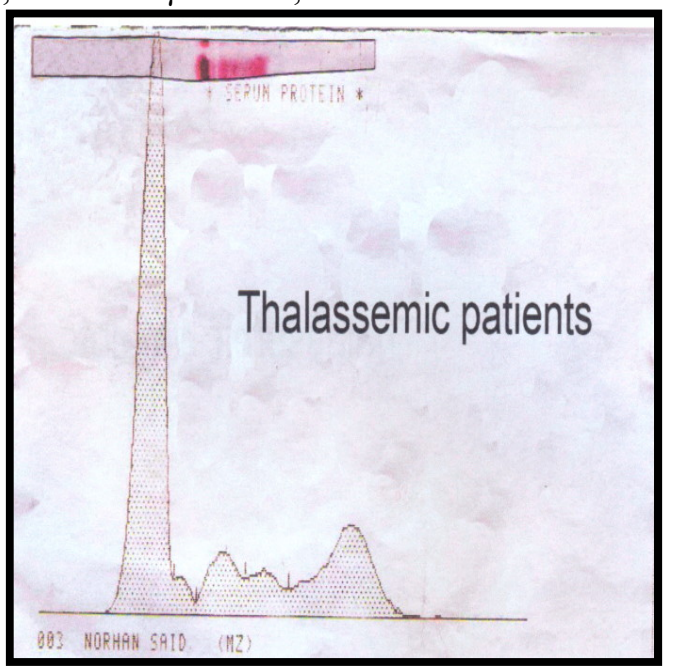

Picture (3):Thalassemia, non hepatic, iron chelating independent

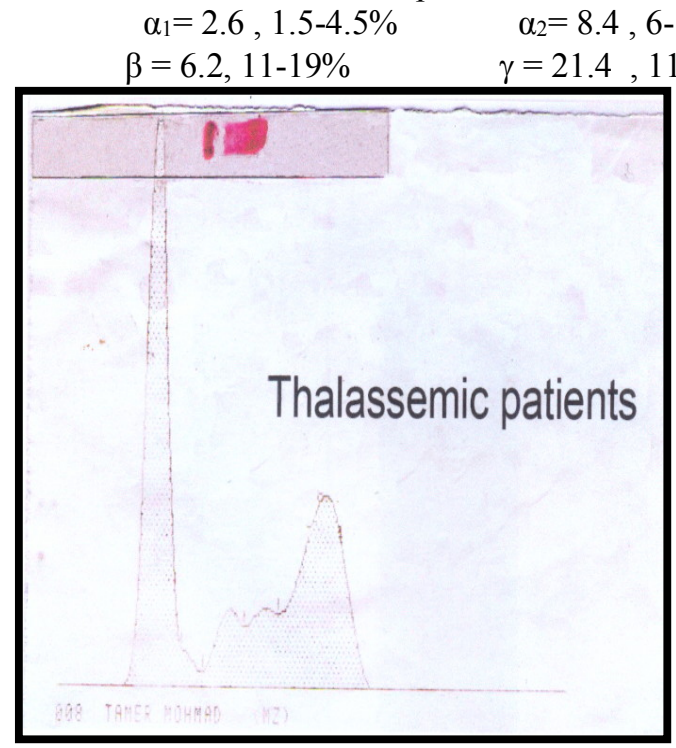

Picture (5): Thalassemia, hepatic , iron chelating dependent $\alpha_{1}=1.8,1.5-4.5 \%$ $\alpha_{2}=7.5,6-12 \%$ $\beta=8.1,11-19 \%$ $\gamma=75.4,11-19 \%$

Plate (1) HPLC hemoglobin variants to evaluate $\mathrm{HbF} \& \mathrm{HBA}_{2}$ 


\section{Hematological parameters changes:}

The RBCs count and indices and other hematological parameters for control and $\beta$ - thalassaemic patients are given in tables 3 and 4. Red blood cells count in vaccinated and non vaccinated groups were lower than that in control groups $(\mathrm{P} \leq$ 0.05). Although vaccinated Thallassimia, hepatitis or non hepatitis, iron chelating dependent or independent had lower RBCs count in relation to non vaccinated groups, the differences were not significant ( table 3 and fig 2). RBCs count was significantly decreased in vaccinated control group compared to non vaccinated control group, but, it was still in human normal value (P $\leq 0.05$ ).

All Thallassimia patient, vaccinated or non vaccinated, hepatitis or non hepatitis, iron chelting dependent or independent had lower $\mathrm{Hb}$ contents than control groups ( vaccinated and non vaccinated, table3).

The Hematocrit $(\mathrm{Ht})$ value in this study revealed that, all Thallassimia patient, vaccinated or non vaccinated, hepatitis or non hepatitis, iron chelting dependent or independent were significantly lower than the control groups ( vaccinated and non vaccinated, table 3).

Table (3) RBCs $\left(\mathrm{x10}^{6} / \mathrm{cm}^{3}\right), \mathrm{Hb}(\mathrm{g} / 100 \mathrm{ml})$ and $\mathrm{Ht}(\%)$ in Thalassemica patients in relation to control groups.

\begin{tabular}{|c|c|c|c|c|c|c|}
\hline \multirow{2}{*}{ Groups } & \multicolumn{2}{|l|}{ RBCs } & \multicolumn{2}{|l|}{$\mathrm{Hb}$} & \multicolumn{2}{|l|}{$\mathrm{Ht}$} \\
\hline & $\begin{array}{l}\text { Non } \\
\text { vaccinated }\end{array}$ & Vaccinated & $\begin{array}{l}\text { Non } \\
\text { vaccinated }\end{array}$ & Vaccinated & $\begin{array}{l}\text { Non } \\
\text { vaccinated }\end{array}$ & Vaccinated \\
\hline Control group & $\begin{array}{l}5.486 \pm \\
0.197\end{array}$ & $\begin{array}{l}4.832 \pm \\
0.141^{*}\end{array}$ & $\begin{array}{l}14.340 \pm \\
0.220\end{array}$ & $\begin{array}{l}12.700 \pm \\
0.529^{*}\end{array}$ & $\begin{array}{l}42.780 \pm \\
0.466\end{array}$ & $\begin{array}{l}37.220 \pm \\
1.584^{* *}\end{array}$ \\
\hline $\begin{array}{l}\text { Thalassemic, } \\
\text { hepatitis, iron } \\
\text { chelating dependent }\end{array}$ & $\begin{array}{l}2.798 \pm \\
0.077^{* *}\end{array}$ & $\begin{array}{l}2.686 \pm \\
0.109^{* *}\end{array}$ & $\begin{array}{l}7.220 \pm \\
0.146^{* *}\end{array}$ & $\begin{array}{l}7.060 \pm \\
0.051^{* *}\end{array}$ & $\begin{array}{l}21.380 \pm \\
0.174^{* *}\end{array}$ & $\begin{array}{l}21.620 \pm \\
0.937^{* *}\end{array}$ \\
\hline $\begin{array}{l}\text { Thalassemic, } \\
\text { hepatitis, iron } \\
\text { chelating independent }\end{array}$ & $\begin{array}{l}3.244 \pm \\
0.134^{* *}\end{array}$ & $\begin{array}{l}2.904 \pm \\
0.087^{* *}\end{array}$ & $\begin{array}{l}8.040 \pm \\
0.136^{* *}\end{array}$ & $\begin{array}{l}7.440 \pm \\
0.361^{* *}\end{array}$ & $\begin{array}{l}24.080 \pm \\
0.962 * *\end{array}$ & $\begin{array}{l}22.660 \pm \\
1.289^{* *}\end{array}$ \\
\hline $\begin{array}{l}\text { Thalassemic, non } \\
\text { hepatitis, iron } \\
\text { chelating dependent }\end{array}$ & $\begin{array}{l}3.318 \pm \\
0.116^{* *}\end{array}$ & $\begin{array}{l}3.124 \pm \\
0.218^{* *}\end{array}$ & $\begin{array}{l}7.54 \pm \\
0.229^{* *}\end{array}$ & $\begin{array}{l}7.960 \pm \\
0.299^{* *}\end{array}$ & $\begin{array}{l}22.260 \pm \\
0.754^{* *}\end{array}$ & $\begin{array}{l}22.900 \pm \\
0.965^{* *}\end{array}$ \\
\hline $\begin{array}{lr}\text { Thalassemic, non } \\
\text { hepatitis, iron } \\
\text { chelating independent }\end{array}$ & $\begin{array}{l}3.370 \pm \\
0.237^{* *}\end{array}$ & $\begin{array}{l}3.230 \pm \\
0.164^{* *}\end{array}$ & $\begin{array}{l}7.225 \pm \\
0.272^{* *}\end{array}$ & $\begin{array}{l}6.950 \pm \\
0.159^{* *}\end{array}$ & $\begin{array}{l}21.375 \pm \\
1.045^{* *}\end{array}$ & $\begin{array}{l}22.567 \pm \\
1.484^{* *}\end{array}$ \\
\hline
\end{tabular}

Results $=\mathrm{M}$ of 5 patients $\pm \mathrm{SE}$

$*=\mathrm{p} \leq 0.05$ when compared with control group (non vaccinated and vaccinated).

$* *=\mathrm{p} \leq 0.01$ when compared with control group (non vaccinated and vaccinated).

Blood indices revealed that significant decreases of MCV values in non vaccinated and vaccinated groups were shown compared to the control group, (except non vaccinated, Thallassimia, hepatitis, either iron chelating dependent or independent) which showed insignificant differences( table 4). Also, non vaccinated, non hepatitis either iron chelating therapy dependent or independent had very high significant decreased MCV $(\mathrm{p} \leq 0.001)$ in relation to vaccinated groups (table 4$)$.

$\mathrm{MCH}$ values in non vaccinated groups were significantly decreased compared to control group , except, Thalassemia, hepatitis, iron chelating therapy dependent which had insignificant difference with control group. Also, non vaccinated Thalassemia, iron chelating independent either hepatitis or non hepatitis had the lowest MCH value than other groups. No significant changes were recorded between vaccinated patients and control group except Thalassemia, non hepatitis, iron chelating independent which had decreased MCH (table 4). 
Table (4): Blood indices MCV (fl), MCH (pg) and MCHC (g/dl) in Thalassemia patients in relation to control groups.

\begin{tabular}{|c|c|c|c|c|c|c|}
\hline \multirow[t]{2}{*}{ Groups } & \multicolumn{2}{|c|}{$\mathrm{MCV}$} & \multicolumn{2}{|c|}{$\mathrm{MCH}$} & \multicolumn{2}{|c|}{$\mathrm{MCHC}$} \\
\hline & $\begin{array}{c}\text { Non } \\
\text { vaccinated }\end{array}$ & Vaccinated & $\begin{array}{c}\text { Non } \\
\text { vaccinated }\end{array}$ & Vaccinated & $\begin{array}{c}\text { Non } \\
\text { vaccinated }\end{array}$ & Vaccinated \\
\hline Control group & $\begin{array}{c}79.400 \pm \\
0.748\end{array}$ & $\begin{array}{c}79.400 \pm \\
1.122\end{array}$ & $\begin{array}{c}27.780 \pm \\
0.483\end{array}$ & $27.440 \pm 0.594$ & $\begin{array}{c}34.760 \pm \\
0.632\end{array}$ & $\begin{array}{c}34.520 \pm \\
0.632\end{array}$ \\
\hline $\begin{array}{l}\text { Thalassemic, } \\
\text { hepatitis, iron } \\
\text { chelating } \\
\text { dependent }\end{array}$ & $\begin{array}{c}76.620 \pm \\
1.593\end{array}$ & $\begin{array}{c}74.800 \pm \\
1.006^{*}\end{array}$ & $\begin{array}{c}26.600 \pm \\
0.416\end{array}$ & $26.260 \pm 0.510$ & $\begin{array}{c}33.920 \pm \\
0.611\end{array}$ & $\begin{array}{c}33.840 \pm \\
1.373\end{array}$ \\
\hline $\begin{array}{l}\text { Thalassemic, } \\
\text { hepatitis, iron } \\
\text { chelating } \\
\text { independent }\end{array}$ & $\begin{array}{c}76.640 \pm \\
1.189\end{array}$ & $\begin{array}{c}75.620 \pm \\
1.057^{*}\end{array}$ & $\begin{array}{c}23.520 \pm \\
0.480^{*}\end{array}$ & $27.400 \pm 0.869$ & $\begin{array}{c}32.340 \pm \\
1.094\end{array}$ & $\begin{array}{c}33.860 \pm \\
0.777\end{array}$ \\
\hline $\begin{array}{c}\text { Thalassemic, non } \\
\text { hepatitis, iron } \\
\text { chelating } \\
\text { dependent }\end{array}$ & $\begin{array}{c}67.780 \pm \\
1.061^{*}\end{array}$ & $\begin{array}{c}75.600 \pm \\
0.639^{*}\end{array}$ & $\begin{array}{c}25.120 \pm \\
1.011\end{array}$ & $27.100 \pm 0.609$ & $\begin{array}{c}33.160 \pm \\
0.700\end{array}$ & $\begin{array}{c}34.200 \pm \\
0.713\end{array}$ \\
\hline $\begin{array}{c}\text { Thalassemic, non } \\
\text { hepatitis, iron } \\
\text { chelating } \\
\text { independent }\end{array}$ & $\begin{array}{c}64.275 \pm \\
0.652^{*}\end{array}$ & $\begin{array}{c}74.717 \pm \\
1.009^{*}\end{array}$ & $\begin{array}{c}24.475 \pm \\
0.636^{*}\end{array}$ & $\begin{array}{c}25.267 \pm \\
0.442 *\end{array}$ & $\begin{array}{c}33.600 \pm \\
0.824\end{array}$ & $\begin{array}{c}35.350 \pm \\
0.448\end{array}$ \\
\hline
\end{tabular}

Results $=\mathrm{M} \pm \mathrm{SE}$

$*=\mathrm{p} \leq 0.05$ when compared with control group (non vaccinated and vaccinated).

$* *=\mathrm{p} \leq 0.01$ when compared with control group (non vaccinated and vaccinated).

No significant changes were recorded in $\mathrm{MCHC}$ in all groups (non vaccinated and vaccinated) compared to control groups (table 4).

White blood cells count (WBCs) elevated in both non vaccinated and vaccinated patients compared to control groups (table 5).

At the same time, a significant decrease $(\mathrm{p} \leq 0.05)$ in Vaccinated Thalassemia, hepatitis, iron chelating dependent compared to non vaccinated Thalassemia, hepatitis, iron chelating dependent (table 5).

Platelet counts in non vaccinated and vaccinated groups were significantly higher than the control groups. Moreover, Thalassemia, hepatitis, iron chelating independent (non vaccinated and vaccinated) recorded the highest count than other groups (table 5).

Table (5) WBCs $\left(\times 10^{3} / \mathrm{cm}^{3}\right)$ and platelets count $\left(\mathrm{x10}^{3} / \mathrm{cm}^{3}\right)$ in Thalassemia patients in relation to control groups.

\begin{tabular}{|c|c|c|c|c|}
\hline \multirow[b]{2}{*}{ Groups } & \multicolumn{2}{|l|}{ WBCs } & \multicolumn{2}{|l|}{ PLts } \\
\hline & $\begin{array}{l}\text { Non } \\
\text { vaccinated }\end{array}$ & Vaccinated & Non vaccinated & Vaccinated \\
\hline Control group & $5.922 \pm 0.049$ & $6.058 \pm 0.402$ & $260.600 \pm 14.102$ & $277.800 \pm 8.907$ \\
\hline $\begin{array}{l}\text { Thalassemic, } \\
\text { hepatitis, iron } \\
\text { chelating dependent }\end{array}$ & $\begin{array}{l}11.138 \pm \\
0.766 * *\end{array}$ & $\begin{array}{l}\text { 8.204 } \pm \\
0.498 *\end{array}$ & $357.800 \pm 24.656 * *$ & $292.400 \pm 29.578 *$ \\
\hline $\begin{array}{l}\text { Thalassemic,hepatiti } \\
\text { s, iron chelating } \\
\text { independent }\end{array}$ & $\begin{array}{l}12.298 \pm \\
0.553 * *\end{array}$ & $\begin{array}{l}13.250 \pm \\
0.315 * *\end{array}$ & $850.200 \pm 33.942 * *$ & $840.600 \pm 29.503 * *$ \\
\hline $\begin{array}{l}\text { Thalassemic, non } \\
\text { hepatitis, iron } \\
\text { chelating dependent }\end{array}$ & $\begin{array}{l}10.248 \pm \\
0.586 * *\end{array}$ & $\begin{array}{l}12.068 \pm \\
0.134 * *\end{array}$ & $357.600 \pm 17.119 * *$ & $414.200 \pm 16.939 * *$ \\
\hline $\begin{array}{lr}\text { Thalassemic, } & \text { non } \\
\text { hepatitis, } & \text { iron } \\
\text { chelating } & \\
\text { independent } & \\
\end{array}$ & $\begin{array}{l}.727 \pm \\
0.276 * *\end{array}$ & $\begin{array}{l}8.727 \pm \\
0.309 *\end{array}$ & $666.750 \pm 23.499 * *$ & $537.333 \pm 31.972 * *$ \\
\hline
\end{tabular}

Results $=\mathrm{M}$ of 5 patients $\pm \mathrm{SE}$

$*=\mathrm{p} \leq 0.05$ when compared with control group (non vaccinated and vaccinated).

$* *=\mathrm{p} \leq 0.01$ when compared with control group (non vaccinated and vaccinated). 
Neutrophils in non vaccinated and vaccinated groups were significantly lower than the control groups. Vaccinated, Thalassemia, non hepatitis, iron chelating independent group recorded the least percent of neutrophils among the vaccinated groups (table 6).

Eosinophil cells count in non vaccinated groups showed no significant correlation among Thalassemia, hepatitis, iron chelating independent; Thalassemia, non hepatitis, iron chelating dependent and control groups. Moreover, Thalassemia, hepatitis, iron chelating dependent group recorded the lowest value and Thalassemia, non hepatitis, iron chelating independent had the highest value of eosinophils than other groups (table 6).

Vaccinated groups showed significant increases of basophile cells count in Thalassemia, hepatitis, iron chelating dependent and independent groups than the control group and all other groups (table 6).

Table (6): Neutrophils, eosinophils and basophils (\%) in Thalassemia patients in relation to control groups.

\begin{tabular}{|c|c|c|c|c|c|c|}
\hline \multirow[t]{2}{*}{ Groups } & \multicolumn{2}{|l|}{ Neutrophils } & \multicolumn{2}{|l|}{ Eosinophils } & \multicolumn{2}{|l|}{ Basophils } \\
\hline & $\begin{array}{l}\text { Non } \\
\text { vaccinated }\end{array}$ & Vaccinated & $\begin{array}{l}\text { Non } \\
\text { vaccinated }\end{array}$ & Vaccinated & $\begin{array}{l}\text { Non } \\
\text { vaccinated }\end{array}$ & Vaccinated \\
\hline Control group & $\begin{array}{l}47.200 \pm \\
0.860\end{array}$ & $\begin{array}{l}50.200 \pm \\
2.177 \\
\end{array}$ & $\begin{array}{l}3.200 \pm \\
0.583 \\
\end{array}$ & $\begin{array}{l}2.800 \pm \\
0.374\end{array}$ & $\begin{array}{l}0.000 \pm \\
0.000 \\
\end{array}$ & $\begin{array}{l}0.000 \pm \\
0.000 \\
\end{array}$ \\
\hline $\begin{array}{l}\text { Thalassemic, } \\
\text { hepatitis, iron } \\
\text { chelating dependent }\end{array}$ & $\begin{array}{l}43.600 \pm \\
2.315^{*}\end{array}$ & $\begin{array}{l}37.400 \pm \\
1.913 * *\end{array}$ & $\begin{array}{l}2.600 \pm \\
0.400\end{array}$ & $\begin{array}{l}6.600 \pm \\
1.029 * *\end{array}$ & $0.4 \pm 0.400$ & $\begin{array}{l}2.600 \pm \\
0.748 * *\end{array}$ \\
\hline $\begin{array}{l}\text { Thalassemic, } \\
\text { hepatitis, iron } \\
\text { chelating } \\
\text { independent }\end{array}$ & $\begin{array}{l}34.200 \pm \\
2.478 * *\end{array}$ & $\begin{array}{l}39.200 \pm \\
0.374 * *\end{array}$ & $\begin{array}{l}3.400 \pm \\
0.872\end{array}$ & $\begin{array}{l}3.600 \pm \\
0.244^{*}\end{array}$ & $\begin{array}{l}1.200 \pm \\
0.734\end{array}$ & $\begin{array}{l}2.600 \pm \\
0.400 * *\end{array}$ \\
\hline $\begin{array}{l}\text { Thalassemic, non } \\
\text { hepatitis, iron } \\
\text { chelating dependent }\end{array}$ & $\begin{array}{l}34.400 \pm \\
0.509 * *\end{array}$ & $\begin{array}{l}39.600 \pm \\
0.678 * *\end{array}$ & $\begin{array}{l}3.000 \pm \\
0.547\end{array}$ & $\begin{array}{l}2.400 \pm \\
0.509 * *\end{array}$ & $\begin{array}{l}1.000 \pm \\
0.632\end{array}$ & $\begin{array}{l}0.400 \pm \\
0.244\end{array}$ \\
\hline $\begin{array}{lr}\text { Thalassemic, } & \text { non } \\
\text { hepatitis, } & \text { iron } \\
\text { chelating } & \\
\text { independent } & \\
\end{array}$ & $\begin{array}{l}37.250 \pm \\
0.750 * *\end{array}$ & $\begin{array}{l}29.833 \pm \\
1.249 * *\end{array}$ & $\begin{array}{l}4.750 \pm \\
0.629 *\end{array}$ & $\begin{array}{l}5.500 \pm \\
0.428 * *\end{array}$ & $\begin{array}{l}1.500 \pm \\
0.288\end{array}$ & $\begin{array}{l}1.000 \pm \\
0.258\end{array}$ \\
\hline
\end{tabular}

Results $=\mathrm{M}$ of 5 patients $\pm \mathrm{SE}$

$*=\mathrm{p} \leq 0.05$ when compared with control group (non vaccinated and vaccinated).

$* *=\mathrm{p} \leq 0.01$ when compared with control group (non vaccinated and vaccinated).

Concerning staff cells count, there was significant increase in non vaccinated groups compared to control group except, Thalassemia, hepatitis, iron chelating dependent which recorded insignificant change. While, there were high significant increases in the staff cells of vaccinated groups except its value in Thalassemia, non hepatitis, iron chelating independent group (table 7).

Lymphocytes increased in all non vaccinated groups and the increase was significant in both Thalassemia, non hepatitis, either iron chelating dependent or independent (table 7). Vaccinated Thalassemia, hepatitis, iron chelating dependent group had high significant decreased lymphocyte count $(\mathrm{p} \leq 0.01)$. While, vaccinated Thalassemia, non hepatitis, iron chelating independent had high significant increased lymphocyte count $(\mathrm{p} \leq 0.01)$ compared to control groups (table 7).

Elevated monocyte counts were recorded in all tested groups ( non vaccinated and vaccinated) compared to control groups. Non vaccinated Thalassemia, hepatitis, iron chelating dependent group had highly significant decrease in monocyte counts compared to vaccinated Thalassemia, hepatitis, iron chelating dependent group $(\mathrm{p} \leq 0.01)$, while, monocyte counts increased significantly $(\mathrm{p} \leq 0.05)$ in non vaccinated Thalassemia, non hepatitis, iron chelating independent compared to vaccinated Thalassemia, non hepatitis, iron chelating independent (table 7). 
Table (7) : Lymphocytes, monocytes and staff cells (\%) in Thalassemia patients in relation to control groups.

\begin{tabular}{|c|c|c|c|c|c|c|}
\hline \multirow[t]{2}{*}{ Groups } & \multicolumn{2}{|c|}{ Lymphocytes } & \multicolumn{2}{|l|}{ Monocytes } & \multicolumn{2}{|l|}{ Staff cells } \\
\hline & $\begin{array}{l}\text { Non } \\
\text { vaccinated }\end{array}$ & Vaccinated & $\begin{array}{l}\text { Non } \\
\text { vaccinated }\end{array}$ & Vaccinated & $\begin{array}{l}\text { Non } \\
\text { vaccinated }\end{array}$ & Vaccinated \\
\hline Control group & $\begin{array}{l}43.600 \pm \\
1.364 \\
\end{array}$ & $\begin{array}{l}41.60 \pm \\
2.768 \\
\end{array}$ & $\begin{array}{l}3.800 \pm \\
0.734 \\
\end{array}$ & $\begin{array}{l}3.400 \pm \\
0.400 \\
\end{array}$ & $\begin{array}{l}2.200 \pm \\
0.200 \\
\end{array}$ & $\begin{array}{l}2.00 \pm \\
0.200 \\
\end{array}$ \\
\hline $\begin{array}{l}\text { Thalassemic, hepatitis, } \\
\text { iron chelating } \\
\text { dependent }\end{array}$ & $\begin{array}{l}46.000 \pm \\
2.607\end{array}$ & $\begin{array}{l}34.400 \pm \\
1.860 * *\end{array}$ & $\begin{array}{l}4.800 \pm \\
1.019\end{array}$ & $\begin{array}{l}10.200 \pm \\
1.113 * *\end{array}$ & $\begin{array}{l}2.600 \pm \\
0.678\end{array}$ & $\begin{array}{l}8.800 \pm \\
0.583 * *\end{array}$ \\
\hline $\begin{array}{l}\text { Thalassemic, hepatitis, } \\
\text { iron chelating } \\
\text { independent }\end{array}$ & $\begin{array}{l}46.800 \pm \\
4.104\end{array}$ & $\begin{array}{l}43.200 \pm \\
0.969\end{array}$ & $\begin{array}{l}9.400 \pm \\
0.927 * *\end{array}$ & $\begin{array}{l}7.600 \pm \\
0.678 * *\end{array}$ & $\begin{array}{l}5.000 \pm \\
0.707 * *\end{array}$ & $\begin{array}{l}3.800 \pm \\
0.200 * *\end{array}$ \\
\hline $\begin{array}{l}\text { Thalassemic, non } \\
\text { hepatitis, iron } \\
\text { chelating dependent }\end{array}$ & $\begin{array}{l}51.400 \pm \\
1.503^{*}\end{array}$ & $\begin{array}{l}47.000 \pm \\
0.837^{*}\end{array}$ & $\begin{array}{l}5.800 \pm \\
0.734^{*}\end{array}$ & $\begin{array}{l}5.600 \pm \\
0.244 * *\end{array}$ & $\begin{array}{l}4.400 \pm \\
0.244 * *\end{array}$ & $\begin{array}{l}5.000 \pm \\
0.316^{* *}\end{array}$ \\
\hline $\begin{array}{l}\text { Thalassemic, non } \\
\text { hepatitis, iron } \\
\text { chelating independent }\end{array}$ & $\begin{array}{l}46.250 \pm \\
0.750^{*}\end{array}$ & $\begin{array}{l}56.167 \pm \\
1.558 * *\end{array}$ & $\begin{array}{l}6.000 \pm \\
0.408 *\end{array}$ & $\begin{array}{l}4.833 \pm \\
0.307^{*}\end{array}$ & $\begin{array}{l}4.250 \pm \\
0.853 * *\end{array}$ & $\begin{array}{l}2.667 \pm \\
0.333\end{array}$ \\
\hline
\end{tabular}

Results $=\mathrm{M}$ of 5 patients $\pm \mathrm{SE}, *=\mathbf{p} \leq \mathbf{0 . 0 5}$ when compared with control group (non vaccinated and vaccinated). $* *=\mathbf{p} \leq \mathbf{0 . 0 1}$ when compared with control group (non vaccinated and vaccinated).

In this study, plasma proteins (Alpha1, Alpha 2, Beta chains and Gamma globulin chain) percentages were determined by electrophoresis and represented in table 8 and plate 2 (pictures 1-5). Alpha1 chain \% increased in both non vaccinated and vaccinated, hepatitis or non hepatitis, iron chelating therapy dependent or independent patients compared to control groups. Also non vaccinated and vaccinated Thalassemia, hepatitis, iron chelating independent had the highest \% of Alphal chain. Regarding to Alpha 2 chain $\%$, the results showed significant increases in different patients groups compared to control groups. On the other hand, Beta chain\% decreased in different patients groups compared to control groups. Evaluation of Gamma chain \% elevated significantly in different patients groups compared to control groups. Moreover, Thalassemia, hepatitis, iron chelating dependent patients detected the highest percentage than all other groups.

Table (8): plasma proteins (Alpha1, Alpha 2, Beta chains and Gamma globulin chain \%) in Thalassemia patients in relation to control groups.

\begin{tabular}{|c|c|c|c|c|c|c|c|c|}
\hline \multirow[t]{2}{*}{ Groups } & \multicolumn{2}{|l|}{ Alpha1 } & \multicolumn{2}{|c|}{ Alpha 2 chain } & \multicolumn{2}{|c|}{ Beta chain } & \multicolumn{2}{|c|}{ Gamma chain } \\
\hline & $\begin{array}{l}\text { Non } \\
\text { vaccinated }\end{array}$ & Vaccinated & $\begin{array}{l}\text { Non } \\
\text { vaccinated }\end{array}$ & Vaccinated & $\begin{array}{l}\text { Non } \\
\text { vaccinated }\end{array}$ & Vaccinated & $\begin{array}{l}\text { Non } \\
\text { vaccinated }\end{array}$ & Vaccinated \\
\hline Control group & $\begin{array}{l}2.420 \pm \\
0.066\end{array}$ & $\begin{array}{l}2.660 \pm \\
0.081\end{array}$ & $\begin{array}{l}8.200 \pm \\
0.114\end{array}$ & $\begin{array}{l}8.540 \pm \\
0.108\end{array}$ & $\begin{array}{l}11.520 \pm \\
0.139\end{array}$ & $\begin{array}{l}11.720 \pm \\
0.124\end{array}$ & $\begin{array}{l}15.220 \pm \\
0.259\end{array}$ & $\begin{array}{l}17.700 \pm \\
0.239^{*}\end{array}$ \\
\hline $\begin{array}{l}\text { Thalassemic, } \\
\text { hepatitis, iron } \\
\text { chelating } \\
\text { dependent }\end{array}$ & $\begin{array}{l}2.820 \pm \\
0.086^{*}\end{array}$ & $\begin{array}{l}2.900 \pm \\
0.084\end{array}$ & $\begin{array}{l}10.340 \pm \\
0.414 * *\end{array}$ & $\begin{array}{l}10.940 \pm \\
0.220 * *\end{array}$ & $\begin{array}{l}.700 \pm \\
0.217^{*}\end{array}$ & $\begin{array}{l}.640 \pm \\
0.117^{*}\end{array}$ & $\begin{array}{l}35.320 \pm \\
0.646^{* * *}\end{array}$ & $\begin{array}{l}30.480 \pm \\
0.606 * * *\end{array}$ \\
\hline $\begin{array}{l}\text { Thalassemic, } \\
\text { hepatitis, iron } \\
\text { chelating } \\
\text { independent }\end{array}$ & $\begin{array}{l}3.600 \pm \\
0.141^{* *}\end{array}$ & $\begin{array}{l}3.580 \pm \\
0.111^{* *}\end{array}$ & $\begin{array}{l}9.740 \pm \\
0.166 * *\end{array}$ & $\begin{array}{l}8.980 \pm \\
0.289 * *\end{array}$ & $\begin{array}{l}.200 \pm \\
0.176^{*}\end{array}$ & $\begin{array}{l}8.220 \pm \\
0.287^{*}\end{array}$ & $\begin{array}{l}30.120 \pm \\
0.290 * * *\end{array}$ & $\begin{array}{l}29.220 \pm \\
0.404 * * *\end{array}$ \\
\hline $\begin{array}{l}\text { Thalassemic, } \\
\text { non hepatitis, } \\
\text { iron chelating } \\
\text { dependent }\end{array}$ & $\begin{array}{l}3.000 \pm \\
0.130 *\end{array}$ & $\begin{array}{l}2.800 \pm \\
0.071\end{array}$ & $\begin{array}{l}9.840 \pm \\
0.129 * *\end{array}$ & $\begin{array}{l}10.520 \pm \\
0.345^{*} *\end{array}$ & $\begin{array}{l}8.260 \pm \\
0.175^{*}\end{array}$ & $\begin{array}{l}9.700 \pm \\
0.179 *\end{array}$ & $\begin{array}{l}16.960 \pm \\
0.441 *\end{array}$ & $\begin{array}{l}16.660 \pm \\
0.213^{*}\end{array}$ \\
\hline $\begin{array}{l}\text { Thalassemic, } \\
\text { non hepatitis, } \\
\text { iron chelating } \\
\text { independent }\end{array}$ & $\begin{array}{l}3.375 \pm \\
0.085 * *\end{array}$ & $\begin{array}{l}2.733 \pm \\
0.092\end{array}$ & $\begin{array}{l}9.550 \pm \\
0.253 * *\end{array}$ & $\begin{array}{l}10.000 \pm \\
0.227 * *\end{array}$ & $\begin{array}{l}8.275 \pm \\
0.193 *\end{array}$ & $\begin{array}{l}8.033 \pm \\
0.161 *\end{array}$ & $\begin{array}{l}17.300 \pm \\
0.367^{*}\end{array}$ & $\begin{array}{l}18.717 \pm \\
0.309 *\end{array}$ \\
\hline
\end{tabular}

Results $=\mathrm{M}$ of 5 patients $\pm \mathrm{SE}, *=\mathrm{p} \leq 0.05$ when compared with control group (non vaccinated and vaccinated). $* *=\mathrm{p} \leq 0.01$ when compared with control group (non vaccinated and vaccinated). $* * *=\mathrm{p} \leq 0.001$ when compared with control group (non vaccinated and vaccinated). 


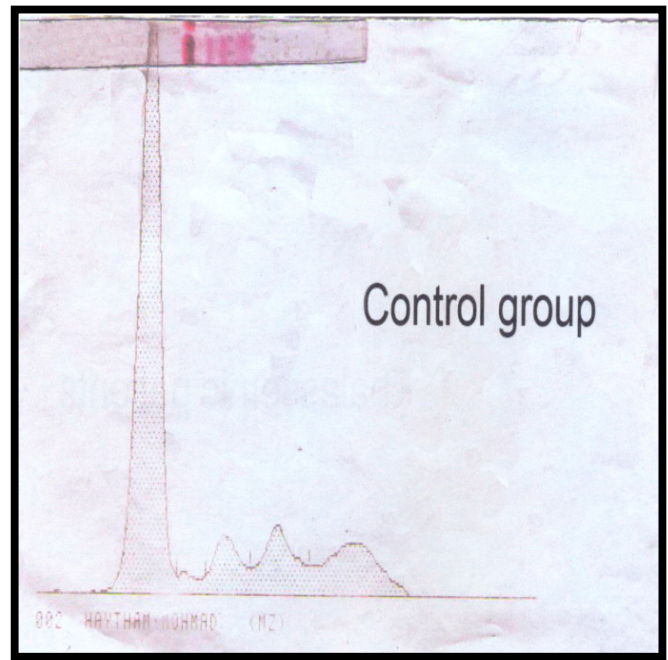

Picture (1): Control group

$\alpha_{1}=2.2,1.5-4.5 \% ; \quad \alpha_{2}=7.8,6-12 \% ; \beta=11.2,11-17 \% \quad \gamma=14.7,11-19 \%$

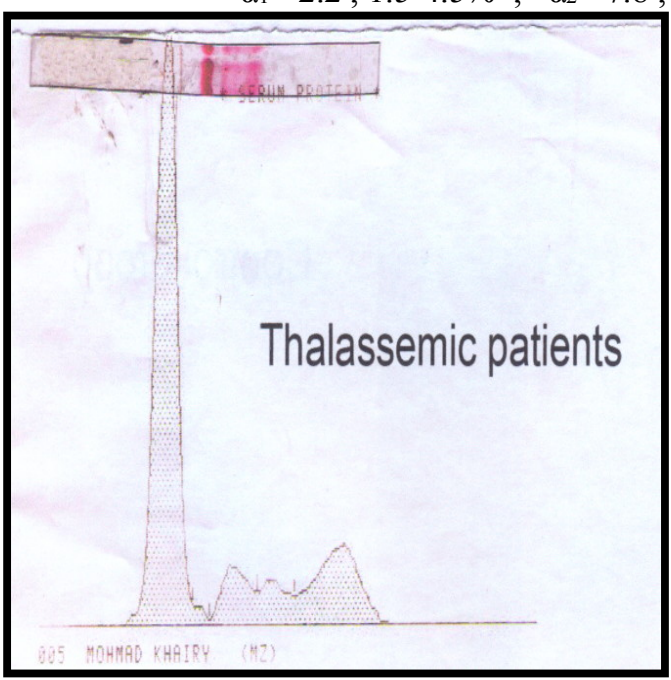

Picture (2): Thalassemia, non hepatic , iron chelating dependent

$\alpha_{1}=1.1,1.5-4.5 \% \quad \alpha_{2}=8.3,6-12 \%$

$\beta=6.0,11-19 \% \quad \gamma=18.1,11-19 \%$

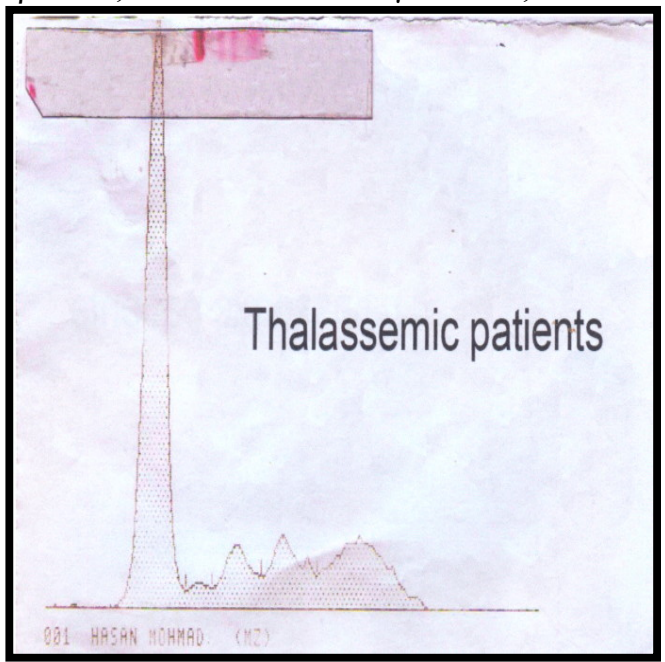

Picture (4):Thalassemia, hepatic , iron chelating independent

$\alpha_{1}=2.4,1.5-4.5 \%$
$\beta=11.4,11-19 \%$

$\alpha_{2}=8.5,6-12 \%$

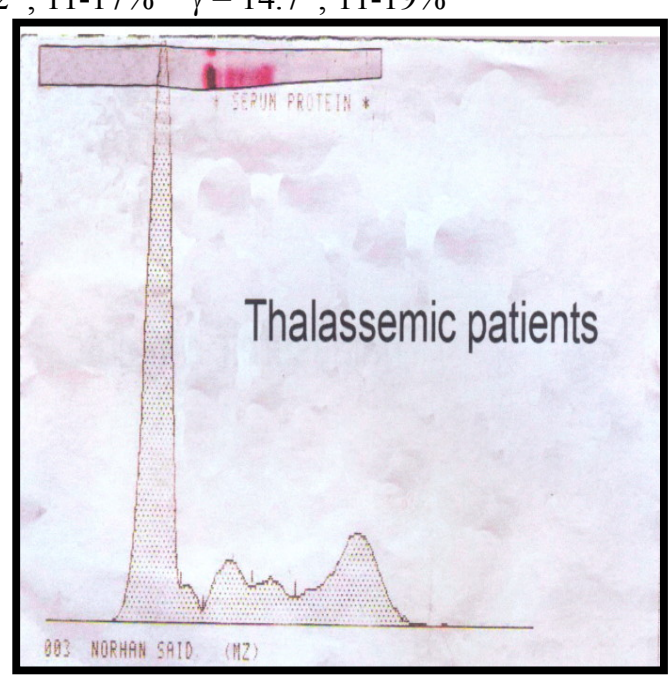

Picture (3): Thalassemia, non hepatic , iron chelating independent

$\alpha_{1}=2.6,1.5-4.5 \% \quad \alpha_{2}=8.4,6-12 \%$

$\beta=6.2,11-19 \% \quad \gamma=21.4,11-19 \%$

\section{1}

Thalassemic patients

Picture (5):Thalassemia, hepatic , iron chelating dependent

$\alpha_{1}=1.8,1.5-4.5 \%$

$\alpha_{2}=7.5,6-12 \%$

$\beta=8.1,11-19 \%$

$\gamma=75.4,11-19 \%$

Plate (2) Protein electrophoresis to evaluate $\alpha, \beta$ and $\gamma$ chains 


\section{Discussion}

Thalassemia major is a worldwide disease, but it is more common in the Mediterranean region, the Middle East, the Asian subcontinent, and southeastern Asia, as well as southwestern Europe and central Africa. It is one of the most common genetic diseases in the world. It is a major health problem, brings much morbidity and early mortality ${ }^{(14)}$.

Thalassemia patients are considered to be one of the high risk groups suffering from post transfusion viral infection such as $\mathrm{HCV}$. In this study, $50 \%$ of patients were +ve for $\mathrm{HCV}$ that was much higher than in the healthy blood control. Also, this percentage is higher than that in Thalassemia patients $(40.5 \%)$ reported in Jordan ${ }^{(6)}$ and (35\%) in Pakistan, ${ }^{(22)}$ but less than the recorded percentage $(63.8 \%)$ in Iran ${ }^{(23)}$.

Several studies with controversial results regarding immunity level and duration of acquired immunity from hepatitis B vaccination have been performed in different countries ${ }^{(21)}$. In a study on children in China, serum anti-HBs was $75 \%$ within 2 years of vaccination and decreased to $48.2 \%, 7$ years post vaccination. In Taiwan, 15 years after the vaccination of neonates, $75 \%$ were anti-HBs positive, but the level was not determined ${ }^{(24)}$. The rate of seropositivity of anti$\mathrm{HBc}$ was $2.9 \%{ }^{(25)}$. This study showed that, $100 \%$ vaccinated, Thalassemia, non hepatitis, iron chelating therapy independent recommended to revaccinate because they had less antibodies against $\mathrm{HBv}$ vaccine compared to the non vaccinated group and that could not be explained.

Ferritin was prognostic at a cut-off of 2,500 $\mathrm{ng} / \mathrm{mL}^{(26)}$. The present study showed that, high levels of serum ferritin have been reported in $\mathrm{HCV}$ - infected patients (1624- 2737ng/mL) vaccinated or non vaccinated, iron chelating therapy dependent or independent. This result is in agreement with Katsanos et al. (10) who recorded increased serum ferritin in all $\beta$ thalassemia patients (range of 213 to 7105 $\mathrm{ng} / \mathrm{mL}$ ). Also, Tabatabaei et al. (2012) found increased serum ferritin in their patients below $2006 \mathrm{ng} / \mathrm{mL}$ responding to ribavirin therapy. Olivieri et al. ${ }^{(27)}$ demonstrated a better prognosis for survival without cardiac disease in transfused patients whose ferritin concentrations remained below $2,500 \mathrm{ng} / \mathrm{mL}$.

Fetal hemoglobin $(\mathrm{HbF})$ is being useful for the diagnosis of $\beta$ - thalassemia syndromes together with the hematological data (mostly $\mathrm{MCV}, \mathrm{MCH})$ and iron status markers ${ }^{(12)}$. In this study, $\mathrm{HbF}$ showed a significant increased values in most groups (12.6- 41.5\%) when compared to control group ( $\mathrm{P}<0.05)$. This result was in agreement with Attia et al. ${ }^{(28)}$ who recorded fetal hemoglobin values in the range (12.5-45.38\%) for $\beta$-thalassemic children. Increased level of $\mathrm{HbF}$, ranging from $10 \%$ to over $80 \%$ is characteristic of homozygous $\beta$-Thallissemia ${ }^{(29)}$.

Quantitative $\mathrm{HbA} 2 \%$ is an important helping factor for the diagnosis of thalassemia. Patients with $\beta$-thalassemia will generally have an increased amount of HbA2 usually (4-6\%) ${ }^{(30)}$. The represented data (table 2) showed significantly elevated $\mathrm{HbA} 2$ values in the non vaccinated and vaccinated specially iron chelating independent either hepatitis or non hepatitis. The noticed decreased HbA2 in iron chelating dependent either hepatitis or non hepatitis may be due to the effect of the iron chelating therapy program compared to iron chelating independent (non vaccinated or vaccinated).

Adult hemoglobin ( $\mathrm{HbA})$ concentration in the present study was significantly decreased in all patients. This can be explained due to elevation of both $\mathrm{HbF} \%$ and $\mathrm{HbA} 2 \%$ in all patients. This result was in agreement with Mosca et al. ${ }^{(30)}$.

Hematological parameters provide information regarding the status of bone marrow activity and hemolysis ${ }^{(31)}$.Decreased RBCs count, $\mathrm{Hb}$ and $\mathrm{Ht}$ (table 3), also MCV and $\mathrm{MCH}$ (table 4) were recorded for all patient groups. This may due to reduced production of RBCs from the bone marrow ${ }^{(28)}$. It was documented that most $\beta$-thalassemic patients suffer from chronic hemolytic anemia because of untimely $\mathrm{RBCs}$ destruction in the bone marrow and spleen (32). They also added that, malformed RBCs number with anisocytosis and poikilocytosis accompanied by hypochromia are well documented features of $\beta$-thalassemia.

The present study showed highly significant increase in the platelet count in all $\beta$ thalassemic patients. The data recorded (table 5) were in agreement with Uggun et al. ${ }^{(33)}$ who reported that thrombocytosis was common in most $\beta$-thalassemic patients.

In this study, white blood cells (WBCs) were slightly elevated or significantly increased in $\beta$-thalassemic patients. Sometimes, the elevated WBCs count may be explained by reveals stippling or ragged inclusion bodies in the red blood cells ${ }^{(34)}$. The differential WBCs in the blood film shifted to the left of neutrophils and neutropenia with relative lymphocytosis. Neutropenia is more common in payients who had not been splenectomized and using iron 
chelating therapy ${ }^{(35)}$. Slightly elevated lymphocytes and agranulocytosis were found in some patients who used iron chelating therapy specially Defriprone therapy ${ }^{(36)}$.

Alphal chain protein was highly increased in patients group that referred to the chronic inflammation which occurred by hepatitis.

The present study showed high significant increase in Alpha 2 chain. Alpha 2 chain is the major constituent of haptoglobin; it is responsible for the binding of hemoglobin released into the circulation when red cells die. Haptoglobin may be elevated, especially during inflammation as part of the acute-phase response ${ }^{(3)}$.

Transferrin comprises the most of Beta band. An increase of Beta -proteins is typical for iron-deficiency anemia due to elevated levels of free transferrin ${ }^{(37)}$. In this study, Beta chain was decreased in all patients group. The reasons of this reduction may be due to the presence of transferrin (the iron bound protein) in $\beta$ - band ${ }^{(38)}$, which is considered as to be the major component of the $\beta$ - globin fraction. Moreover, it agrees with previous study of Al-Mustansiriya (39).

hemoglobinopathies

The various immunoglobulin classes (IgG, $\operatorname{IgA}, \operatorname{IgM}, \operatorname{IgD}$ and IgE) are usually of Gamma band. Gamma-globulins increase in inflammatory disorders like chronic infections (e.g. viral hepatitis) ${ }^{(34)}$. This rises in $\gamma$ - globulin levels were also demonstrated in patients with sickle cell anemia and genetic hemochromatosis by Rivero et $\mathrm{al}^{(40)}$ and Fargion ${ }^{(41)}$ respectively. On the other hand, the $\gamma$-globulin band consist of C- reactine protein, which is elevated as mush as (1000) fold in response to inflammation, also the acute nature of the inflammatory syndrome as assessed by CRP concentration was confirmed by high level of production of IgM and IgG antibodies ${ }^{(42,43)}$.

The recommendations from this study are: 1- All thalassemia patients or their parents should receive information regarding the risk of viral infections associated with blood transfusion and other routes.

2- All thalassemia patients should receive hepatitis B immune globulin (HBIG) and hepatitis B vaccine immediately before first blood transfusion.

\section{References}

1- Omar A, Abdel Karim E, Gendy WE, Marzouk I and Wagdy M (2005): Molecular basis of $\beta$-thalassemia in Alexandria, Egypt. J. Immunol., 12:15-24.
2- Desouky OS, Selim, NS, El-Barawy EM and EL-Marakby SM (2009): Biophysical characterization of $\beta$-thalassemic red blood cells. Cell. Biochem. Biophys., 55, 45-53.

3- El- Beshlawy A, Kaddah N, Omar N (2005): Experience with the oral iron chelator Deferiprone singl and compined with Deferoxamine. $9^{\text {th }}$ Internat, Conference on Thalassemia.

4- Batebi A, Pourreza A and Esmailian RR (2012): Discrimination of beta-thalassemia minor and iron deficiency anemia by screening test for red blood cell indices. Turk. J. Med. Sci., 42 (2): 275-280.

5- Duman H, Canatan D, Alanoglu G, Sutçu R and Nayır T (2013): The Antıxidant Effects of Capparis Ovata and Deferasirox in Patients with Thalassemia Major. J. Blood Disorders Transf., 4 (3): 142- 146.

6- Al Shayyab M, Baticha A, El Khateeb M (2001): The prevalence of hepatitis $B$, hepatitis $C$ and human immunodeficiency virus markers in multi-transfusion patients. J. Trop. Pediatr., 47(4):239-42.

7- Younus M, Hassan K, Ikram,E, Naseem L, Zaheer HA and Khan MF (2004): Hepatitis C Virus Seropositivity in Repeatedly Transfused Thalassemia Major Patients. Internat. J. Path., 2(1):20-23.

8- Tabatabaei SV Alavian SM Keshvari M Behnava B, Miri SM Elizee PK Zamani F, Kafiabad SA, Gharehbaghian A, Hajibeigy B and Lankarani KB (2012): Low Dose Ribavirin for Treatment of Hepatitis C Virus Infected Thalassemia Major Patients; New Indications for Combination Therapy. Hepat. Mon., 12(6): 372381.

9- Prati D (2000): Benefits and complications of regular blood transfusion in patients with betathalassemia major. Vox-Sang., 79(3): 129-137

10- Katsanos KH, Chaidos A, Christodoulou DK, Tzambouras N, Zervou E, Bourandas KL, and Tsianos EV (2005): Epidemiological and clinical characteristics of $\mathrm{HCV}$ infection in transfusiondependent thalassemia. Annals Gastroenterol., 18(1): 54-56.

11- Chakravarti A, Verma V, Kumaria $R$ and Dubey AP (2005): Anti- HCV seropositivity among multiple transfused patients with beta thalassemia. Indian. Med. Assoc., 103 (2): 6466.

12- Mosca A Paleari R, Leone $D$ and Ivaldi G (2009): The relevance of hemoglobin $F$ measurement in the diagnosis of thalassemias and related hemoglobinopathies. Biochem., 42: 17971801.

13- Birgens H, Ljung R (2007): The thalassemia syndromes. Scan. J. Clin. Lab. Invest., 67:11-26.

14- Kharbanda WP, Modak WS, Singh WPS and Garg GRK (2002): Beta thalassaemia trait fitness for fighter flying: Two case reports. Ind. J. Aerospace Med., 46(1): 75-82. 
15- Farmakis D Giakoumis A and Polymeropoulos E (2003): Pathogenetic aspects of immune deficiency associated with betathalassemia. Med. Sci. Monit., 9(1): A19-22.

16- Walker EM Jr, Walker SM (2000): Effects of iron overload on the immune system. Ann. Clin. Lab. Sci., 30: 354-365

17- Fong TL, Han SH and Tsai NC(1998): A pilot randomized, controlled trial of the effect of iron depletion on long- term response to alpha- inter forming patients with chronic hepatitis C. J. Hepat., 28: 369-374.

18- Galanello R (1995): Evaluation of an automatic HPLC analyzer for Thalassemia and hemoglobin variant screening,J. Auto. Chem., 17(2): 73-76.

19- Dacie JV and Lewis SM (1995): Practical hematology. Churchil Livingstone, 1995. Bain, B.J. Blood cells- practical guide. $2^{\text {nd }}$ ed. Blackwell Science.

20- Brown BA (1984): Hematology: Principles, practices and procedures. $4^{\text {th }}$ ed. Lea and Fibiger. Philadelphia; 300: 32-38.

21- Sharifi Z, Milani $S$ and Shooshtari MM (2010): Study on Efficacy of Hepatitis B Immunization in Vaccinated Betathalassemia Children in Tehran. Iran J. Pediatr., 20 (2):211215.

22- Rehman M and Lodhi Y (2004): Prospects of future of conservative management of $\beta$ thalassemia major in a developing country. Pak J Med Sci., 20(2): 105-107.

23- Ansar $M$ and Kooloobandi A (2002): Prevalence of hepatitis $\mathrm{C}$ infection in thalassemia and hemodialysis patients in north Iran-Rasht. J Viral Hepat., 9(5):390-2.

24- Li H, Li RC and Liao SS (1998): Persistence of hepatitis $\mathrm{B}$ vaccine immune protection and response to hepatitis $\mathrm{B}$ booster immunization. World J. Gastroenterol., 4 (6): $493-$ 6.

25- Ni YH, Chang MH and Huang LM(2001): Hepatitis B virus infection in children and adolescents in a hyperendemic area: 15 years after mass hepatitis B vaccination. Ann Intern Med., 135(9):796-800.

26- Borgna -Pignatti C, Rogolotto $S$ and De Stefano $P$ (1998): Effecacy of defroxamine in preventing complications of iron overload in patients with Thalassimia major. N. Engl. J. Med., 331: 567.

27- Olivieri NF Nathan DG, MacMillan JH, Wayne AS, Liu PP and McGee A (1994): Survival in medically treated patients with homozygous b-thalassemia. N. Engl. J. Med., 331:574-8.

28- Attia MMA Sayed AM, Ibrahim FA, Mohammed AS and El-Alfy MS (2011): Effects of antioxidants vitamins on some hemoglobin properties and erythrocytes in
Homozygous $\beta$-Thalassemia . Rom. J. Biophys., 21(1): 1-16.

29- Rees DC (2000): Hemoglobin F and hemoglobin E/ $\beta$-thalassemia. J. Pediatr. Assoc., 103(2): 64- 66 .

30- Mosca A, Paleari R, Ivaldi G, Galanello R and Giordano PC (2008): The role of haemoglobin A2 testing in the diagnosis of thalassaemias and related haemoglobinopathies. J Clin Pathol., 62:13-7.

31- Iniaghe OI, Egharevba $O$ and Oyewo EB (2013): Effect of Aqueous Leaf Extract of Acalypha wilkesiana on Hematological Parameters in Male Wistar Albino Rats. British J. Pharmaceutical Res., 3(3): 465-471

32-Pattanapanyasat K, Noulsri E, Fucharoen S, Lerdwana S, Lamchiagdhase $P$, Siritanaratkul $N$ and Webster HK (2004): Flow cytometric quantitation of red blood cell vesicles in Thalassemia.Clin. Cytometry, 57b: 23.

33- Uggun, A, Kadayifei, A and Erein, N (2000): Interferon treatment for thrombocytopenia associated with HCV infection. Int. J. Clin. Pract., 54(10): 683.

34-Ernest BT (2001): Williams Hematology. 6th ed. New York: McGraw- Hill,Dis. 27: 294.

35- Maggio A, D'Amico G, Morabito A, Capra M and Ciancialli $P$ (2002): Defriprone versus defroxaminelinical in patients with Thalassemia major: A randomized clinical trial. Blood cells Mol. Dis., 28: 196-208.

36- Fosburg M.T, Han SH and Tsai NC (1999): Treatment of Cooleys anemia. Blood, 76: 435444.

37-Vavricka SR, Burri E, Beglinger C, Degen L and ManzSerum $M$ (2009): Protein Electrophoresis: An Underused but Very Useful Test. Digest., 79: 203-210.

38- Muola A (2001): Clinical biochemical study about oxidation and antioxidant in patients with non insulin dependent diabetes mellitus. M. D. Thesis. College of Education of (Ibn- Al-haitham) University of Baghdad.

39- Al-Mustansiriya I (2006): Study of Protein profile in patients with $\beta$ - thalassemiaJ. Sci., 17 (4): 120- 135.

40- Rivero RA, Macias C and Aranda RE (1991): Immunologic changes in sickle cell anemia. Sangre., 36(1): 15-20.

41- Fargion S, Mandelli C, and Piperno A (1992): Survival and prognostic factors in $2 \mathrm{R}$ Italian patients with genetic hemochromatosis hepatology. 15(4): 655-9.

42- Murray RK, Granner DK, Rodwell VW (2003): Harper's Biochemistry, 27th Edn., Appleton and Lang, California.

43- Bishop ML, Pody EP and Schoff L (2005): "Clinical chemistry principles, Procedures, correlations" 5th ed., pp: 105-106, 194-212. Lippincott Williams \& Wilkins. 